\title{
Annotation
}

\section{Congenital heart disease in the adolescent}

Congenital heart disease occurs in approximately 1 in 100 births. Left to nature $60 \%$ would die in infancy, $25 \%$ in the newborn period, and probably only $15 \%$ would survive to adolescence and adult life. ${ }^{1}$ Since Gross and Hubbard (1938) ligated the duct, ${ }^{2}$ Craaford and Nylin (1945) resected coarctation of the aorta, ${ }^{3}$ and Blalock and Taussig (1945) with their shunt changed the fortunes of children with cyanotic congenital heart disease, ${ }^{4}$ surgical treatment has advanced so that most lesions can be repaired or palliated and no age is too young for cardiac surgery. Whether some of the newer procedures for hypoplastic left heart and other complex malformations are advances or retrograde steps in terms of suffering and survival remain to be evaluated.

Challenged by the surgeon, the art and science of preoperative diagnosis and care developed. As the major problems presented in infants and neonates, paediatricians were inevitably involved. Thus the specialty of paediatric cardiology was born and grew in strength to maturity, celebrated in its own World Congress held in London in 1980 where the problems of adolescents were specifically addressed in the final plenary session on Adolescent SurvivorsTriumphs or Disasters? ${ }^{5}$ The prospect for survival to adult life to become a wage earner and enjoy family life is now available for the child born with serious congenital cardiac anomalies.

Is the concept of 'total correction' really true? In terms of 'excellent' and good results, do these patients need care, support, or further surgical treatment when they reach adolescence* or adulthood? The answer is that many do even though many lead normal lives. ${ }^{6}$ How many 'grown up' people with congenital heart disease are there in the United Kingdom and how many need help or further surgery? Certainly they are increasing in number each year.

The National Heart Hospital in 1976 extended inpatient facilities to cope with increasing numbers of adolescent cardiac patients by opening a paediatric and adolescent unit. These young patients usually like to be with their own age group.

\footnotetext{
*For convenience and ease this refers to patients aged 12-19 years but there are no absolute limits for the start or finish of adolescence! It is different for girls and boys.
}

Depending on sexual maturity and preferences some may wish to be in adult wards. It is surprising that many of the young adults into their 20 s or 30 s prefer to stay with the young rather than be with the older adult cardiac patients. The National Heart Hospital has accepted the commitment to the growing up of congenital heart patients and established a service for their special problems both as inpatients and outpatients, for long distance national consultations, and for further specialised surgical expertise. A total of $94 \%$ of adolescent cardiac admissions have congenital heart disease and $75 \%$ have had cardiac surgery in childhood or infancy.

Hospital beds continue to be needed for adolescents with cardiological problems. Arrhythmias precipitate the need for admission most commonly in the medical patients and this includes recurrent pacemaker changes. Pacing may be difficult because of shortage of venous access or odd anatomy. Arrhythmias in this group are often complex requiring expert management and electrophysiological knowledge and investigation. Care is required not to administer drugs that cause deterioration in myocardial function of the already abnormal ventricle upon which systemic output depends. Endocarditis, progressive pulmonary vascular disease, myocardial dysfunction, and effects of lung infections require inpatient treatment. The diagnosis and referral of infective endocarditis is frequently late, not only in adolescents but also in older patients, not because of delay in consultation but because of lack of thought of the diagnosis by practitioners. Aware of the need for prevention in a 'card carrying' or scarred young patient, doctors continue to prescribe antibiotics for non-specific symptoms without appropriate prior blood tests and cultures. This results in delayed diagnosis and blood culture negative endocarditis with a worse prognosis and greater destruction than if the diagnosis had been correctly sought and confirmed early. This is inexcusable in those with known congenital heart disease, who have already been operated on, and where the risks are clearly spelt out. Adolescents may be reluctant to consult doctors. After a serious complication they often bypass local help and come directly to the centre where they feel 'their hearts are understood'. The service has been designed so that patients and their 
physicians can easily make telephone contact and through the unit data base, with about 3500 patients indexed and followed up, there is instant information available. This has helped in the management of many questions and difficulties relating to depression, threatened suicide, prison sentences, unwanted pregnancy, demands of unusual life style, and employment without having to first seek notes.

In the last five years demands for adolescent cardiac beds have changed; there is less demand now for invasive investigation, which has been replaced by non-invasive outpatient investigation, but there is more demand for interventional procedures and assessment for heart and heart/lung transplantation. This is a courageous group of young people, costly to investigate and maintain, and demanding of many support services. Looking after them while they are waiting for a heart presents many medical problems as well as social problems and demands for psychological support, which must be addressed by the adolescent/young adult unit once the assessment is completed.

The cardiac surgeon's skills are needed for reoperations, particularly to replace valves and conduits used in the initial 'correction' (better considered as radical repairs). Natural valves, particularly the aortic valve, may degenerate and calcify after earlier valvotomy or become critically narrow in the second decade. Complex congenital defects, previously helped by extracardiac palliation, damage by endocarditis, and residual lesions claim surgical attention. There are different problems than at the first operation and parents are often unprepared for the need, morbidity, and fatalities of reoperation. Interventional techniques may reduce some of the surgical needs but still the reoperation risks are increased and should be dealt with by the most experienced and cautious. Too many deaths have occurred during reopening by an unwary or inexperienced junior surgeon. The surgical management of 'grown up' congenital heart disease urgently requires rationalisation. It is not for the general adult cardiac surgeon to 'have a go'.

These problems in the adolescent cardiac patients continue in the adult 'until 70'. The problems of acquired ischaemic heart disease and hypertension are added to the abnormal heart with each added decade. In addition, the requirements of these young patients with congenital heart disease for advice on life's normal problems such as contraception, employment, insurance, prison confinement, marriage, pregnancy, and risks of affected offspring, and whether it is safe for them to hold an 'until 70' driving licence or vocational licence are a demanding part of outpatient work. Special advice is often needed about extracardiac surgery, trauma, and accidents. When advice is not sought, nor given by the informed, the patient's life may be at risk.

\section{Who knows and who cares?}

The problems of the adolescent with congenital heart disease continue into adult life. The National Heart Hospital statistics show not only an increase in adolescent referrals but also a rise in inpatient admissions of patients over 20 and 30 years with problems related to treated congenital heart disease. It should be accepted that most of these patients require someone with knowledge to continue their long term supervision and be available for consultation in respect of the problems that confront teenagers and adults. ${ }^{7}$ Perloff is facing the same problems in the United States and is trying to establish a programme for the care of these patients. ${ }^{8}$ There are some patients with treated lesions such as ligated ducts (also sectioned ducts), closed atrial septal defects, and most of those with mild pulmonary valve stenosis and pulmonary valvotomy in whom regular or even any informed supervision is unnecessary. In most treated congenital heart disease, particularly the cyanotic and complex, good medical supervision is important for their maintained well being. Even simple atrial septal defects may develop fast atrial rhythm disorders 10-20 years after the successful surgery and this can carry an embolic risk.

Paediatricians and paediatric cardiologists must accept the need not only to make proper referral arrangements but also to indoctrinate the patient and family about importance of continuing informed medical supervision. The age when it is necessary to refer to a 'grown up' clinic varies according to the cardiological demands, local expertise, and maturity of the patient. The time must be recognised when the child no longer wants the tripartite relationship with parent and paediatrician but wants the one to one adult relationship with the doctor. This problem often causes resentment with refusal to attend clinics. Adolescents are naturally rebellious in their pursuit of independence. Whoever or wherever the patient is cared for, it must be realised that it is for longer than adolescence and includes the life span. Having accepted the need, the arrangements and implementation are sometimes difficult. At the present time flexibility is vital.

In some centres with close proximity (and good relations) between paediatric and adult cardiologists, joint clinics can be organised. Many, including the patient, feel the paediatric cardiologist should continue the care but often it is not practical, as he or she does not have access to adult beds or knowledge of adult acquired heart diseases. On the 
other hand, the cardiologist trained in coronary and valvular disease is ignorant and even fearful of congenital heart disease, particularly when complex. There may be particular problems when the paediatric cardiac surgical centre is in a children's hospital that is separate in location and in philosophy from its adult counterpart. Forward looking arrangements must be organised as growing up is certain for childhood successes and survivors. Just as the need for treatment of neonatal and infant cardiac patients in specialist supraregional centres was recognised by the Department of Health and Social Security, ${ }^{9}$ so I believe special arrangements should be made for the grown up congenital heart patients. There will soon be more of these than infants.

The ROCK philosophy must be implemented:

Recognise that there is a problem that requires attention and that most physicians are ignorant in this field.

Organise formal follow up and supervision for patients by someone or a group who are familiar and interested.

Centralise patients with informed cardiologists and surgeons experienced in the problems of congenital heart disease. A supraregional concept may be necessary in planning, preferably where there are designated inpatient facilities for the young to stay together. They usually like it and prefer to be with young rather than with older cardiac cases involving failure, strokes, etc.

Knowledge is vital. Special education and training, but not in neonates, is important. Experience in reoperation and basic anatomy and pathology of congenital heart disease is mandatory for surgeons involved.

The problems of long term care must apply to other childhood diseases. Paediatricians must accept that their successful treatment has produced a new medical community beyond the age of their special- ist competence. It should not be difficult to solve once the problem is recognised, provided consultants are not rigid in their intellectual practices and are willing to share their skills and facilities. These patients have been through much in cost, time, endeavour, and courage. They deserve to be maintained as productive members of society. Today's problems may be different from tomorrow's but it is certain there will be as many or more into $2000 \mathrm{AD}$ until the occurrence of congenital cardiac and cardiovascular malformations is reduced or even abolished. Regions, supraregions, and even the Department of Health could usefully address the problem -it has much wider implication than for the survivors of congenital heart disease.

\section{References}

' MacMahon B, McKeown T, Record RG. The incidence and life expectation of children with congenital heart disease. $\mathrm{Br}$ Heart J 1953;15:121-9.

2 Gross RE, Hubbard JP. Surgical ligation of a persistent ductus arteriosus. JAMA 1939;112:729.

${ }^{3}$ Craaford C, Nylin G. Congenital coarctation of the aorta and its surgical treatment. Journal of Thoracic Surgery 1945;14:34i-61.

4 Blalock A, Taussig $H$. The surgical treatment of malformations of the heart. JAMA 1945;128:189-202.

5 Somerville J. Adolescent survivors-triumphs or disasters? In: Godman MJ, ed. Paediatric cardiology. Vol 4. Edinburgh: Churchill Livingstone, 1981:756-67.

6 Perloff JK. Pediatric congenital cardiac becomes a postoperative adult. The changing population of congenital heart disease. Circulation 1973;47:606-19.

${ }^{7}$ Somerville J. Congenital heart disease in adults and adolescents. Br Heart J 1986;56:395-7.

${ }^{8}$ Perloff JK. The UCLA adult congenital heart disease program. Am J Cardiol 1986;57:1190-2.

9 Third Report of a Joint Cardiology Committee of the Royal College of Physicians of London and The Royal College of Surgeons. Provision of services for the diagnosis and treatment of heart disease in England and Wales. Br Heart J 1985;53:47782.

J Somerville

National Heart Hospital, Westmoreland Street, London W1M $8 B A$ 